\title{
HACIA UNA FiLOSOFÍA DE LA LENGUA VULGAR. FILOSOFÍA, POESÍA Y TRADUCCIÓN EN EL CONVIVIO DANTESCO
}

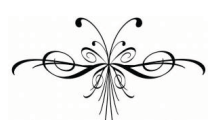

MARIANo PÉREZ CARRASCO*

Resumen: En este artículo estudio los vínculos que se establecen entre la filosofía, la poesía y la traducción en la primera obra filosófica de Dante: el Convivio. Sostengo la tesis de que la filosofía dantesca en el Convivio está fuertemente condicionada por la elección de la lengua vernácula. Dado que a comienzos del siglo XIV el volgare toscano no era una lengua comúnmente utilizada en la escritura de obras filosóficas, Dante tuvo que justificar su elección lingüística, i.e. se vio obligado a probar los recursos filosóficos de la lengua vernácula. Por ese motivo, Dante escribió no sólo una obra filosófica en vulgar, sino también una filosofía de la lengua vulgar.

Luego de unas palabras introductorias, el artículo despliega sus argumentos en cinco capítulos, cuyos temas abordan: el nuevo ideal de la filosofía sostenido por Dante; $(\S 1)$; la tarea de traducir tanto la filosofía cuanto la verdad: la primera, del latín a la lengua vernácula; la segun$\mathrm{da}$, del sentido literal (falso) al sentido alegórico (verdadero) ( $§ 2$ ); el modo en que la esencia filosófica de la lengua vernácula es actualizada -en sentido aristotélico- por la prosa dantesca ( $§ 3)$; el modo en que el pensamiento dantesco se transfiere desde la poesía hacia la filosofía ( $\S 4)$; por último, como conclusión, las reflexiones de Dante acerca de la imposibilidad de traducir poesía (§ 5).

Palabras clave: Dante; Convivio; filosofía; poesía; traducción

\begin{abstract}
This paper aims at showing the links philosophy, poetry and translation establish in Dante's first philosophical work, the Convivio. I sustain the thesis that Dante's philosophy at the stage of the Convivio is strongly conditioned by the choice of vernacular language. Given that at the beginning of the $14^{\text {th }}$ Century Tuscan vernacular was not a language commonly used in the writing of philosophical works, Dante felt obliged to justify his choice - i.e., he had to prove the philosophical resources of the vernacular. That is why he wrote not just a philosophical work in vernacular, but also a philosophy of vernacular language.

After a few introductory words, the paper displays its arguments in five chapters, concerning: Dante's new ideal of philosophy $(\S 1)$; the task of translating both philosophy and truth: the first, from Latin to vernacular, and the second from literal (false) to allegorical meaning (true) $(\S 2)$; the way in which the philosophical essence of vernacular language is actualized - in an Aristotelian sense by Dante's prose ( $\S 3$ ); the way in which Dante's thought passes from poetry to philosophy ( $\$ 4)$; and, as a conclusion, Dante's reflections on the impossibility of translating poetry $(\S 5)$.
\end{abstract}

Keywords: Dante; Convivio; philosophy; poetry; translation 


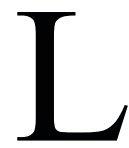

a pregunta por el origen de las ideas filosóficas - no ya sólo por su contenido- ha adquirido una creciente legitimidad en el ámbito filosófico desde fines del siglo XIX. Esta pregunta, explícitamente formulada en la Genealogía de la moral, constituye uno de los puntos centrales del pensamiento de Nietzsche ${ }^{1}$. A partir de Nietzsche, la investigación del origen, la búsqueda de la genealogía de las ideas filosóficas, constituye uno de los filones del pensamiento contemporáneo. Similar habría sido la posición de Husserl en la Philosophie der Arithmetik, a juzgar por la crítica de Frege, quien, en una reseña a ese libro, señala, contra las tesis husserlianas, que es preciso conocer qué es el mar del Norte, no cómo surge en nosotros la noción del mar del Norte ${ }^{2}$. En la primera mitad del siglo XX, por último, Ortega y Gasset argumenta acerca del valor fílosófico no sólo de los conceptos en sí mismos, sino también del origen de esos conceptos. Según Ortega, no basta con comprender qué cosa sea el mar del Norte, es también necesario conocer cómo se llega a producir en nosotros el concepto del mar del Norte. Una filosofía que no tenga en cuenta la génesis de sus propios conceptos es -en opinión de Ortegauna "fillosofía ingenua": "Entiendo por filosofía ingenua o injustificada toda aquella que se deja fuera de su cuerpo doctrinal los motivos que llevan a ella, es decir, que no considera como porción constitutiva de la filosofía misma todo lo que ha inducido al hombre a esa creación filosófica" (J. Ortega y Gasset, 1959, p. 40). Según esta perspectiva, el pensamiento dantesco, en especial en el Convivio, no sería una filosofía ingenua: en él están explícitamente tematizados e incorporados a la filosofía misma los motivos que llevaron a su autor a escribir esta obra. Esos motivos, que fueron expresados por Dante en el proemio del Convivio, no deben ser relegados por el dantista a los márgenes de la filosofía del poeta, sino que deben ser considerados como lo que de hecho son: componentes centrales de esa filosofía.

En este artículo estudio los argumentos que Dante presenta en favor de la necesidad de escribir filosofía en prosa vernácula, esto es, en una lengua que carecía de tradición filosófica. Esos argumentos involucran las relaciones que la filosofía y la poesía establecen, mediante el expediente de la traducción, con la verdad. En un primer capítulo introductorio expongo el nuevo ideal de la filosofía presente en el Convivio $(\S 1)$. El ideal filosófico que Dante desarrolla en el Convivio supone una doble traducción: por un lado, la traducción de la filosofía del latín al volgare; por otro lado, la traducción del sentido literal al sentido alegórico. Esta doble traducción tiene por fin llevar la filosofía a un ámbito que le era ajeno (la ciudad, no ya las universidades y los monasterios) y a un público que le era extraño (los illitterati). Este proceso es estudiado en el segundo capítulo (§ 2). En el tercer capítulo muestro cómo Dante al escribir el Convivio realiza la finalidad de la lengua vernácula, i.e., actualiza la esencia filosófica de la lengua vulgar (§3). El capítulo cuarto estudia el modo y los motivos por los que

\footnotetext{
* Esta investigación fue desarrollada en el marco de una beca otorgada por el Consejo Nacional de Investigaciones Científicas y Técnicas (Conicet) de la República Argentina. El autor es Doctor en Filosofía por la Universidad de Buenos Aires, donde se desempeña como Auxiliar Docente en la cátedra de Historia de la Filosofía Medieval.

${ }^{1}$ F. Nietzsche (1997, p. 61): “¿quiere alguien mirar un poco hacia abajo, al misterio de cómo se fabrican ideales en la tierra?". Subrayado del Autor. Este texto es citado como epígrafe por L. Bianchi (1990, p. 149).

${ }^{2}$ Cf. G. Frege, "Edmund Husserls Philosophie der Arithmetik", en Zeitschrifta für Philosophie der Arithmetik, CIII, 1894. (Apud R. Raggiunti, 1986, pp. 14-15).
} 
el pensamiento dantesco cumple la traducción de la poesía a la filosofía (§ 4). Por último, el quinto capítulo recupera los temas de la traducibilidad de la poesía y de la posibilidad de expresión de la verdad poética por la prosa filosófica $(\S 5)$.

\section{Un nuevo ideal de la filosofía}

El libro primero del Convivio constituye un caso particularmente elocuente de surgimiento de un nuevo ideal de la filosofía. Este ideal recoge las características más sobresalientes del tipo de intelectual aparecido en el siglo XIII entre los maestros de artes parisinos, para quienes la filosofía no habría constituido tanto un saber profesional cuanto un modo de vida ${ }^{3}$. Lo específico del ideal dantesco reside en que introduce un cambio en la concepción de la actividad filosófica, que implica asimismo una modificación del público al que los productos de esa actividad van dirigidos: el público de la filosofía deja de ser exclusivamente el mundo universitario y pasa a ser la sociedad en su conjunto, aquellos a los que Dante llama los illitterati, pues ignoran el latín, esto es, las litterae $^{4}$. Estos cambios aparecen de un modo paradigmático en el primer capítulo del Convivio ${ }^{5}$. Allí Dante construye una ética que es heredera del eudemonismo intelectualista de la Ética a Nicómaco y toma de ella rasgos marcadamente aristocráticos y elitistas. Sin embargo, el proyecto filosófico formulado en el proemio de ese texto plantea la intención de llevar a cabo una profunda democratización del saber. De modo que se presenta la siguiente paradoja: por un la-

\footnotetext{
${ }^{3}$ Cf. L. Bianchi (1990), quien ha estudiado la cuestión del status philosophi en los siglos XIII y $\mathrm{XIV}$, y la creciente autoconsciencia de los intelectuales en aquel período. Siguiendo una perspectiva muy cercana a Bianchi, A. De Libera (1991), ha discutido los supuestos básicos del estudio de J. Le Goff (1965). Bianchi cita el Convivio Dante cuando describe la polémica acerca de la autonomía de la filosofía como disciplina y como modo de vida. Dante -dice Bianchi- excluye de la categoría de filósofos a "“li legisti, li medici e quasi tutti li religiosi, che non per sapere studiano ma per acquistare moneta o dignitade" (Conv. III.xi.10). La polemica epistemologica volta a rivendicare alla filosofia un suo spazio specifico, una sua autonomia contro una diffusa teoria e pratica di subordinazione, si traduceva così sul piano etico-sociale nell'esaltazione di una vita che superbamente si rinchiudeva e pretendeva di esaurirsi nell'orizzonte della contemplazione razionale" (p. 158).

${ }^{4}$ Litteratus hace referencia a aquel que sabe leer y escribir, pero dado que al menos desde el siglo IV -señala Mariken Teeuwen- la distancia entre la lengua latina escrita y la lengua hablada era enorme, el término pasó a significar "una persona que sabe latín”, y, luego, ya que quienes sabían latín, esto es, los litterati, eran clerici, ambos términos acabaron por identificarse, de modo que el correspondiente illitterati pasó a referir a los laici. Cf. M. Teeuwen (2003, sub voce "litteratus, illitteratus", pp. 92-94).

${ }^{5}$ La tesis de que el Convivio de Dante encarna un nuevo ideal que a la vez continúa y rompe con el modelo "universitario" de los magistri parisinos ha sido defendida por A. De Libera, y constituye la texis central de su Penser au Moyen Âge, que De Libera resume del siguiente modo: "Il $\mathrm{y}$ a deux sotres d'intellectuels au tournant du XIIIe siècle et du XIVe siècle: ceux qui inventent l'existence philosophique à partir des textes et ceus qui tentent de vivre cette vie en incarnant les métaphores du discours magistral. En outre, si les grands centres de déprofessionnalisation de la philosophie sont urbains, les villes où l'existence philosophique a tenté de s'organiser, notamment Cologne, n'avaient pas d'université, mais seulement des couvents de formation (studia) appartenant aux ordres mendiants: les intellectuals du second type ne sont donc pas des universitaires, mais des marginaux. Lus ensembles, ces deux aspects de la "nouvelle culture urbaine" nous livrent l'essentiel d'un mécanisme plus général: la laïcisation de la pensée et le passage à la langue vernaculaire, ici le moyen haut allemand, là 1'”italien", quelquefois le français. Les médiateurs de l'ideal philosophique ont parlé vulgaire, ils ont nom Dante pour l'Italie, Meister Eckhart pour l'Allemagne" (p. 13).
} 
do, una ética para la cual sólo unos pocos hombres, en virtud de sus propios méritos, son capaces de acceder a la verdadera felicidad; por otro lado, el Convivio -es decir, el texto en el cual esta ética está contenida- se propone como una vulgarización (volgarizzazione), una popularización de ese mismo conocimiento a través del cual se alcanza la felicidad ${ }^{6}$. La paradoja se vuelve aún más dramática al constatar que ambos términos - popularización y elitismo- dependen del mismo ideal de vida feliz. Si la reflexión se detiene en este plano exclusivamente ético, la paradoja parece ser irresoluble. Por eso la ética de Dante, su concepción de la vita beata, ha encontrado su resolución en un proyecto político -el Imperium universale - que concilia y sintetiza los términos de la paradoja señalada, pues el buen orden político sienta las condiciones necesarias para la plena realización de la humanitas en su conjunto ${ }^{7}$. Al establecer una paz duradera y estable, la política permite que una mayor cantidad de hombres alcancen el fin que les es propio: pensar, es decir, actualizar la potencialidad intelectiva. El proyecto político dantesco se presenta, pues, como la conclusión del ideal filosófico dantesco, según el cual el objetivo de la filosofía consistiría en realizar un novedoso ideal de humanitas $^{8}$ (Kantorowicz, 1985).

El nuevo ideal de la filosofía presentado por Dante en el Convivio muestra una síntesis entre la ética elitista de los maîtres ès arts parisinos y una tendencia popularizadora, democratizante, que se manifiesta en primer lugar en la elección del vehículo para transmitir esa ética, esto es, en la elección de la lengua vernácula. De este modo, en el Convivio se ponen en escena las tensiones culturales propias de comienzos del Trecento, tanto en el ámbito de la filosofía cuanto en el de la literatura. Esta tensión entre aristocratismo y popularización se verifica en el incipit de la obra:

Como dice el Filósofo al comienzo de la Primera Filosofía, todos los hombres naturalmente desean saber. La razón de ello puede ser y es que toda cosa, impulsada por la providencia, se inclina hacia su propia perfección en virtud de su propia naturaleza; por eso, dado que la ciencia es la última perfección de nuestra alma, en la cual se encuentra nuestra felicidad última, todos estamos naturalmente sujetos a desearla 9 .

Aquí Dante no hace más que señalar un lugar común del aristotelismo: todos los hombres poseen una tendencia (desiderio) natural al conocimiento. Pero en un segundo momento pone en evidencia los motivos por los que, aunque

\footnotetext{
${ }^{6}$ Esta tensión entre aristocratismo y popularización que se verifica en el ámbito del discurso filosófico, también puede constantarse en el ámbito del discurso literario. Mario Marti ha señalado las dos tendencias que se enfrentan en la cultura literaria de la época, y cabe destacar que Dante -con su tensón con Forese Donati y sus rimas para la dama Pietra, por ejemplo- ha participado de ambas (M. Marti, 1974, p. 125).

${ }^{7}$ Cf. Conv. IV.iv-ix. Excepto indicación, todas las traducciones me pertenecen.

${ }^{8}$ De allí el carácter marcadamente práctico que tienen las dos obras filosóficas dantescas: por un lado, una ética fundada en una concepción antropológica, desarrollada en el Convivio; por otro lado, una teoría política que se funda en aquella ética, y que es expuesta en unos pocos capítulos del libro cuarto del Convivio (IV.iv-ix) y en la Monarchia. De este modo, ambas obras aparecen como inescindibles, pues se enmarcan dentro del mismo proyecto filosófico.

${ }^{9}$ Conv. I.i.1: "Sì come dice lo Filosofo nel principio de la Prima Filosofia, tutti li uomini naturalmente desiderano di sapere . La ragione di che puote essere ed è che ciascuna cosa, da providenza di propria natura impinta, è inclinabile a la sua propria perfezione; onde, acciò che la scienza è ultima perfezione de la nostra anima, ne la quale sta la nostra ultima felicitade, tutti naturalmente al suo desiderio semo subietti”.
} 
este deseo de conocer es universal, sin embargo no todos los hombres pueden acceder al conocimiento por todos deseado: "Muchos están privados de esta nobilísima perfección por diversas razones que, dentro y fuera del hombre, lo alejan del hábito de la ciencia" 10 . Por una parte, malformaciones corporales impiden recibir adecuadamente los principios del conocimiento: un ciego es incapaz de comprender los colores de una pintura; un mudo, de ejecutar el canto. "Nuestro intelecto se puede decir sano y enfermo [...]. Se puede decir que es sano cuando su operación no está impedida por ninguna mala disposición del ánimo o del cuerpo"11. Luego de enumerar las posibles corrupciones del alma, procede a enumerar los impedimentos corporales: "la mente puede no ser sana por alguna enfermedad o defecto del cuerpo: bien por defecto de algún principio desde el nacimiento, como en los enfermos mentales; bien por una alteración del cerebro, como en los frenéticos" ${ }^{\prime 2}$. No es a estos enfermos a los que está dirigido el Convivio, pues sus mentes no son libres, y en consecuencia no están bien dispuestos para recibir la luz de la verdad ${ }^{13}$. Tampoco está dirigido a aquellos que persiguen "deleites viciosos, en los cuales se encuentra tanto engaño que, gracias a ellos, tiene[n] toda cosa por vil" 14 , ni a los perezosos que habiendo nacido en un lugar geográfico y en un ambiente social poco propicios para el estudio no han sido capaces de ponerse en marcha para buscar el conocimiento. El público al que se dirige el Convivio está compuesto por aquella inmensa mayoría de hombres y mujeres, que, por tener que "ocuparse del cuidado familiar y civil [...], no pueden encontrarse en un ocio de especulación"15. El público al que Dante se dirige está constituido por burgueses, por hombres que se ocupan de sus negocios y de sus familias, y por ciudadanos, hombres ocupados en los asuntos de la res publica. Unos y otros (bien pueden coincidir en la misma persona) no tienen impedimentos físicos ni anímicos para recibir el conocimiento; su único impedimento es la falta de tiempo, que les ha impedido adquirir el estudio de la gramática -es decir, del latín-, obligada puerta de acceso a la cultura.

El proyecto filosófico que Dante elabora está orientado, pues, a satisfacer un deseo natural (el hambre y la sed de conocimiento) que no puede ser sa-

\footnotetext{
${ }^{10}$ Conv. I.i.2: "Veramente da questa nobilissima perfezione molti sono privati per diverse cagioni, che dentro all'uomo e di fuori da esso lui rimovono dall'abito di scienza".

${ }^{11}$ Conv. IV.xv.11: "Onde è da sapere che lo nostro intelletto si può dir sano e infermo: e dico "intelletto" per la nobile parte dell'anima nostra che con uno vocabulo "mente" si può chiamare. Sano dire si può quando per malizia d'animo o di corpo impedito non è nella sua operazione; che è conoscere quello che le cose sono, sì come vuole Aristotile nel terzo dell'Anima".

${ }^{12}$ Conv. IV.xv.17: "E secondo malizia o vero difetto di corpo, può essere la mente non sana: quando per difetto d'alcuno principio dalla nativitade, sì come sono mentecatti; quando per l'alterazione del cerebro, sì come sono frenetici".

${ }^{13}$ Conv. IV.xv.18: "Per che a quelli intelletti che per malizia d'animo o di corpo infermi non sono liberi, espediti e sani a la luce de la veritade, dico essere manifesto l'oppinione de la gente, che detto è, essere vana, cioè sanza valore".

${ }^{14}$ Conv. I.i.3: "Dentro dall'uomo possono essere due difetti e impedimenti: l'uno dalla parte del corpo, l'altro dalla parte dell'anima. Dalla parte del corpo è quando le parti sono indebitamente disposte, sì che nulla ricevere può, sì come sono sordi e muti e loro simili. Dalla parte dell'anima è quando la malizia vince in essa, sì che si fa seguitatrice di viziose dilettazioni, nelle quali riceve tanto inganno che per quelle ogni cosa tiene a vile".

${ }^{15}$ Conv. I.i.4: "Di fuori dall'uomo possono essere similemente due cagioni intese, l'una delle quali è induttrice di necessitade, l'altra di pigrizia. La prima è la cura familiare e civile, la quale convenevolemente a sé tiene delli uomini lo maggior numero, sì che in ozio di speculazione essere non possono. L'altra è lo difetto del luogo dove la persona è nata e nutrita, che tal ora sarà da ogni studio non solamente privato, ma da gente studiosa lontano".
} 
tisfecho por la mayoría de los hombres debido a causas externas: la falta de tiempo libre. El Convivio opera una traducción del lenguaje filosófico del latín al vulgar, con el fin de llevar a cabo una "vulgarización" de la filosofía. El sentido de esta traducción está orientado a satisfacer un deseo natural que permanece insatisfecho por la ausencia de libros escritos en lengua materna ${ }^{16}$. En este punto, el proyecto de Dante constituye una novedad de la que su autor es plenamente consciente:

\begin{abstract}
Manifiestamente, pues, puede ver quien bien considera, que quedan pocos que puedan arribar al hábito por todos deseado, y son casi innumerables los impedidos que viven siempre hambrientos de este alimento. ¡Oh, beatos aquellos pocos que se sientan a la mesa donde se come el pan de los ángeles, y míseros aquellos que comparten el alimento con las ovejas ${ }^{17}$ ! Pero dado que todo hombre es naturalmente amigo de todo hombre, y todo amigo se duele del defecto de aquel a quien ama, aquellos que se alimentan en tan alta mesa no carecen de misericordia hacia los que ven que, en pastura para bestias, van comiendo hierba y bellotas. Y dado que la misericordia es madre de beneficio, siempre aquellos que saben ofrecen liberalmente su buena riqueza a los verdaderos pobres, y son como una viva fuente, con cuya agua se refresca la sed natural que más arriba se ha nombrado. Y yo, pues, que no me siento a la beata mesa, pero que, habiendo huido de la pastura del vulgo, a los pies de los que se sientan recojo lo que de ellos cae, y conozco la mísera vida de aquellos que he dejado atrás, por la dulzura que siento en lo que poco a poco recojo, movido por la misericordia, sin olvidarme, para los míseros he reservado alguna cosa [las canciones doctrinales], que ya he expuesto, hace algún tiempo, a sus ojos; y con eso los he vuelto mayormente deseosos [de saber]. Por lo cual ahora, queriendo prepararlos, pretendo realizar un convivio general de aquello que les he mostrado, y del pan que es necesario para un tal alimento, sin el cual no podría ser comido por ellos ${ }^{18}$.
\end{abstract}

El período se estructura como un silogismo práctico, con su premisa mayor universal ("todo hombre es naturalmente amigo de todo hombre"), su premisa menor particular ("yo [...] que conozco la mísera vida de aquellos que

\footnotetext{
${ }^{16}$ Acerca del volgare como lengua materna, cf. De vulg. eloq. I.i.2: "Vulgarem locutionem appellamus eam qua infantes adsuefiunt $\mathrm{ab}$ adsistentibus, cum primitus distinguere voces incipiunt: vel, quod brevius potest, vulgarem locutionem asserimus, quam sine omni regula, nutricem imitantes, accipimus".

${ }^{17}$ Cf. H. de Lubac, "Bovinus intellectus", en (Idem, 1961, pp. 113-128).

${ }^{18}$ Conv. I.i.6-11: "Manifestamente adunque può vedere chi bene considera, che pochi rimangono quelli che all'abito da tutti desiderato possano pervenire, e innumerabili quasi sono li 'mpediti che di questo cibo sempre vivono affamati. Oh beati quelli pochi che seggiono a quella mensa dove lo pane delli angeli si manuca! e miseri quelli che colle pecore hanno comune cibo! Ma però che ciascuno uomo a ciascuno uomo naturalmente è amico, e ciascuno amico si duole del difetto di colui ch'elli ama, coloro che a così alta mensa sono cibati non sanza misericordia sono inver di quelli che in bestiale pastura veggiono erba e ghiande sen gire mangiando. E acciò che misericordia è madre di beneficio, sempre liberalmente coloro che sanno porgono della loro buona ricchezza alli veri poveri, e sono quasi fonte vivo, della cui acqua si refrigera la naturale sete che di sopra è nominata. E io adunque, che non seggio alla beata mensa, ma, fuggito della pastura del vulgo, a' piedi di coloro che seggiono ricolgo di quello che da loro cade, e conosco la misera vita di quelli che dietro m'ho lasciati, per la dolcezza ch'io sento in quello che a poco a poco ricolgo, misericordievolemente mosso, non me dimenticando, per li miseri alcuna cosa ho riservata, la quale alli occhi loro, già è più tempo, ho dimostrata; e in ciò li ho fatti maggiormente vogliosi. Per che ora volendo loro apparecchiare, intendo fare un generale convivio di ciò ch'i' ho loro mostrato, e di quello pane ch'è mestiere a così fatta vivanda, sanza lo quale da loro non potrebbe essere mangiata. Ed ha questo convivio di quello pane degno, con tale vivanda qual io intendo indarno non essere ministrata".
} 
he dejado atrás") y su consecuencia práctica ("pretendo realizar un convivio general"). El contenido de la argumentación desarrolla distintas variantes de metáforas alimenticias aplicadas al conocimiento: todos lo desean pero muchos viven hambrientos (deseo / hambre); pocos se sientan a la mesa donde se come el pan de los ángeles, pero muchos comparten su alimento con las bestias (ángeles / bestias) (Bianchi, 1994); los verdaderos pobres poseen sed de saberes, los pocos ricos son fuentes que sacian esa sed (riqueza / pobreza; sed / fuente). Estas oposiciones, que son el núcleo retórico-conceptual del pasaje, encuentran su centro y se resuelven de un modo original en la figura del poeta-filósofo -la primera persona del singular- que cumple la función angélica de mediador entre quienes carecen de conocimiento y viven en el hambre ("quelli che in bestiale pastura veggiono erba e ghiande sen gire mangiando") y aquellos que "se sientan a la beata mesa" y comen "el pan de los ángeles" ("quelli pochi che seggiono a quella mensa dove lo pane delli angeli si manuca" $)^{19}$.

Dante señala aquí que son "pocos los que pued[e]n arribar al hábito por todos deseado, y son casi innumerables los impedidos que viven siempre hambrientos de este alimento" 20 . Su función como autor del Convivio consiste en llevar ese alimento deseado a la mayor cantidad de hombres. El aspecto cuantitativo de esta empresa filosófica es subrayado al final del libro primero:

Es tiempo, pues, de suministrar los alimentos. Este será aquel pan de cebada con el cual se saciarán miles, y a mí me sobrarán las canastas llenas. Este [pan] será luz nueva, nuevo sol, que surgirá allí donde el usado se ponga, y dará luz a aquellos que se encuentran en tinieblas de oscuridad porque el usado sol a ellos no alumbra (subr. prop.) $)^{21}$.

Volvamos ahora a la figura del narrador (“a mí me sobrarán...”). Toda narración encuentra su eje en la figura del narrador, que le imprime a los hechos narrados una determinada perspectiva. En el Convivio, el narrador-personaje tiene características muy peculiares: su autoridad no reside en haberse sentado a la beata mesa de la sabiduría, sino en haber pertenecido al amplio círculo de los verdaderos pobres ("habiendo huido de la pastura del vulgo"), y, ahora, es decir, en el momento cero, en el instante bautismal en que comienza la escritura, recoge las migajas de los que poseen la sabiduría para entregársela a aquellos pobres que no pueden siquiera sentarse a los pies de la mesa, pues carecen del tiempo y del conocimiento necesarios para ello. Estamos ante un ideal plebeyo de la filosofía, que comienza a competir con el monopolio universitario y clerical de la cultura (Bertelloni, 2008, pp. xxi ss.).

Este procedimiento argumentativo (afirmar la propia superioridad sobre la base de una inferioridad) es característico de la filosofía dantesca (Gilson, 1937). El poeta-filósofo no adquiere su autoridad tanto de lo que conoce cuanto de la doble consciencia de lo que desconoce y de lo que desconocen aquellos a quienes se dirige, pero que - esto sí- él conoce. Este último conocimiento, que

\footnotetext{
${ }^{19}$ Esta figura "mediadora", angélica en el sentido de aquel que comunica entre dos órdenes, ha sido atribuida a Dante por A. De Libera (1991, p. 24), en discusión con Le Goff.

${ }^{20}$ Conv. I.i.6.

${ }^{21}$ Conv. I.xiii.11-12: "Tempo è d'intendere a ministrare le vivande. Questo sarà quello pane orzato del quale si satolleranno migliaia, e a me ne soverchieranno le sporte piene. Questo sarà luce nuova, sole nuovo, lo quale surgerà là dove l'usato tramonterà, e darà lume a coloro che sono in tenebre ed in oscuritade, per lo usato sole che a loro non luce".
} 
pertenece enteramente al orden de la experiencia biográfica ("conozco la vida mísera de aquellos que he dejado atrás"), es el que finalmente autoriza a Dante a escribir el Convivio: un banquete para pobres.

Un último punto a tratar es el motivo que impulsa a Dante a transmitir las "migajas" que caen de la beata mesa. Este motivo es una característica del objeto mismo -i.e., del conocimiento, metafóricamente: el pan, las migajas-. Se trata de la dulzura (dolcezza). El conocimiento es, ante todo, algo dulce, cuya dulzura se va descubriendo poco a poco ("la dulzura que siento en lo que poco a poco recojo"). Para comprender el sentido y la importancia de esta característica atribuida al conocimiento, es necesario prestar atención a las metáforas que Dante pone en juego. El alimento, es decir, el conocimiento, es la verdad, objeto de la fílosofía, no la filosofía misma. Pero el alimento-conocimiento en el que reside la verdad son las canciones. La filosofía es el pan que acompaña y hace digeribles las canciones. Al igual que en la Biblia, también en el Convivio la verdad se muestra en versos, y, como veremos, Dante es consciente de la dificultad que la traducción poética representa para la comprensión y transmisión del sentido del texto (Toynbee, 1921, p. 51-52). En el Convivio, la verdad se da en el poema. La filosofía ayuda a clarificar esa verdad que aparece oculta bajo el manto de las imágenes, las rimas y demás "vestimentas" poéticas: he aquí la función hermenéutica de la filosofía.

Se comprende ahora el extraño lugar que Dante se atribuye a sí mismo en el proceso de transmisión del conocimiento. Él es el poeta que ha presentado al pueblo (il volgo), "hace algún tiempo", algunas canciones, dulces al oído pero ásperas en el sentido ${ }^{22}$, es decir, de difícil comprensión. Los poemas encienden el deseo de saber, pero no lo satisfacen, por eso precisan un comentario, del mismo modo en que la lectura de la Biblia, en la teología medieval, es iluminada gracias al comentario, que aclara su sentido, es decir, que permite digerir el alimento. Así como Dante se sienta a los pies de "la beata mesa", así "cualquiera que por el cuidado familiar o civil ha permanecido en el hambre humana",23 está invitado a sentarse a la mesa de este convivio, es decir, de este banquete para aquellos que han quedado excluidos de la mesa principal, y a los pies de esta mesa pobre han de sentarse los perezosos, "pues no son dignos de un más alto

\footnotetext{
${ }^{22}$ Dulcis es término retórico, cuyo contrario es asper. En Dante el uso es claro; así reza, por ejemplo, el incipit de la rima 46 (CIII): “Cosí nel mio parlar voglio esser aspro". En el Convivio: "Le dolci rime d'amor ch'i' solìa", donde sostiene que para hablar de la virtud (valore) es preciso una rima áspera y sutil ("rima aspr'e sottile"). En De vulg. eloq. I.x.2, Dante afirma que su amigo Cino da Pistoia y él mismo son quienes han poetizado de un modo más dulce y sutil en toscano ("qui dulcius subtilisque poetati vulgariter sunt [...], puta Cynus Pistoriensis et amicus eius"). En la Commedia, Dante se dirige a Guido Guinizzelli confesándole que la admiración que siente por él toma su origen en "li dolci detti vostri" (Purg. XXVI, 112), pues Guinizzelli ha compuesto rimas de amor "dolci e leggiadre" (ib., 99); y, en el famoso pasaje en el que Dante bautiza a la escuela poética de su juventud, Bonagiunta da Lucca confiesa que nunca ha alcanzado el dulce estilo nuevo que escucha en los nuevos poetas (“"' $\mathrm{O}$ frate, issa vegg'io", diss'elli, "'il nodo / che '1 Notaro e Guittone e me ritenne / di qua dal dolce stil novo ch'i'odo!").

${ }^{23}$ Conv. I.i.13: "Ma vegna qua qualunque è per cura familiare o civile nella umana fame rimaso, e ad una mensa colli altri simili impediti s'assetti; e alli loro piedi si pongano tutti quelli che per pigrizia si sono stati, ché non sono degni di più alto sedere: e quelli e questi prendano la mia vivanda col pane che la farà loro e gustare e patire".
} 
asiento" 24 . Unos y otros, dice Dante, "tomen mi alimento [las canciones] con el pan [el comentario filosófico], el cual les hará gustar y digerir el alimento"25.

En el siguiente capítulo, Dante enuncia la estructura que habrá de tener el banquete. Catorce canciones de contenido doctrinal y amoroso ("sì d'amor come di vertù"), serán sometidas a interpretación filosófica. Estos comentarios son necesarios pues sin ellos las canciones aparecían oscuras ("aveano d'alcuna oscuritade ombra"), de modo que pocos eran capaces de comprender su contenido (bontade). Los comentarios serán filosóficos, no estéticos; su función consistirá en echar luz sobre el verdadero significado (sentenza) de los versos:

El alimento de este convivio será ordenado de catorce maneras, es decir, ca-
torce canciones cuya materia será tanto el amor cuanto la virtud, las cuales sin
el presente pan tenían la sombra de cierta oscuridad, de modo que a muchos
les agradaba más su belleza que su bondad. Pero este pan, es decir, la presente
exposición, será la luz que hará manifiesto todo el color de su significado (sen-
tenza $)^{26}$.

El juego metafórico continúa. Los poemas-alimento, canciones doctrinales acerca del amor y la virtud, que Dante ya ha hecho públicas ("ya he expuesto, hace algún tiempo, a sus ojos"), se presentaban oscuros en su sentido; por ese motivo sus primeros lectores, seducidos por la bellezza, dejaban escapar aquello de lo que esa belleza era medio, esto es, su bondad (bontade). Por eso es necesario servir nuevamente el alimento, pero, para evitar una "mala digestión", es decir, una mala comprensión del significado, esta vez las canciones irán acompañadas de un comentario en prosa ("la presente exposición", "el presente pan"). Aquí el eje conceptual vuelve a ser una metáfora: este comentario no sólo es el pan que permite la digestión, sino la luz que debe hacer que los colores del sentido aparezcan a los ojos.

Siguiendo una larga tradición alegorista (Pépin, 1971, Hollander, 1969, Singleton, 1958), Dante atribuye a cada canción dos sentidos principales: el sentido que aparece primero, de un modo superficial, es decir, en la literalidad de las palabras, y el sentido más profundo, alegórico, al cual sólo la reflexión puede abrir camino, interpretando de otro modo (állēi agoréuō) el primer sentido literal. La verdad de lo dicho no reside en la superficie de lo dicho, sino en aquello que, no dicho, está contenido en las palabras. Esto último es la alegoría. Esta verdad alegórica es la que debe ser puesta en evidencia, traída a la luz ("farà parvente"), por los comentarios en prosa. Pero en ningún momento hay que olvidar que esa verdad se encontraba ya, de un modo original, en las palabras poéticas, aunque allí no de un modo explícito, sino bajo "la sombra de cierta oscuridad" ("d'alcuna oscuritade ombra") ${ }^{27}$. El vínculo entre la verdad, la alegoría y la literalidad es expuesto por Dante al final del capítulo comentado:

\footnotetext{
${ }^{24} \mathrm{Ib}$.

${ }^{25} \mathrm{Ib}$.

${ }^{26}$ Conv. I.i.14-15:" La vivanda di questo convivio saràe di quattordici maniere ordinata, cioè di quattordici canzoni sì d'amor come di vertù materiate, le quali sanza lo presente pane aveano d'alcuna oscuritade ombra, sì che a molti loro bellezza più che loro bontade era in grado. Ma questo pane, cioè la presente disposizione, sarà la luce la quale ogni colore di loro sentenza farà parvente".

${ }^{27}$ Cf. Conv. I.i.14-15.
} 
Y dado que mi verdadera intención es otra que la que por fuera muestran las canciones antedichas, mediante una exposición alegórica pretendo mostrarlas, luego de discutir la historia literal; de modo que una y otra razón darán sabor a aquellos que están convidados a esta mesa ${ }^{28}$.

En resumen, asumiendo su carácter de mediador entre la verdad pura (la teología y la filosofía) y la pura ignorancia (el vulgo), Dante proyecta una obra capaz de transmitir a los ignorantes algo de aquella sabiduría a la que él mismo no tiene pleno acceso. Para llevar a cabo esa transmisión del conocimiento hacia los illitterati, Dante debe realizar una empresa novedosa: escribir filosofía en una lengua sin tradición filosófica.

\section{Del latín al vulgar, de la letra a la verdad}

Una vez determinado que el público al que esta obra se dirige son los illitterati, Dante pasa a justificar la elección del medio con el cual se dirige a ellos. Justifica así el uso filosófico de la lengua vernácula. Este es uno de los aspectos más novedosos de la obra, y Dante, una vez más, parece ser plenamente consciente de la innovación que está llevando a cabo. Esta consciencia lo lleva a emplear un amplio arsenal argumentativo tendiente a fundamentar la necesidad del empleo de la lengua vernácula en los comentarios a sus canciones.

Entre los motivos que inclinan a Dante al uso del vulgar se encuentra la mayor "liberalidad" (liberalitade) que de este modo adquieren el autor y la obra; $\mathrm{y}$, dado que la liberalidad es una virtud, al escribir el Convivio en lengua vulgar, obra y autor se volverán más virtuosos:

Aún más, el vulgar dará un regalo no solicitado, que el latín no habría dado: pues se dará a sí mismo como comentario, no habiendo sido jamás solicitado por ninguna persona; y esto no se puede decir del latín, que ya fue solicitado muchas veces como comentario y como glosa de diversos textos, tal como se puede ver claramente en los comienzos de muchos. Y así es manifiesto que una acabada liberalidad me impusló al vulgar antes que al latín ${ }^{29}$.

El latín era la lengua en que estaban escritos tanto los comentarios cuanto los textos comentados. Estos textos-objeto formaban la constelación de las auctoritates. Sus auctores habían pasado la prueba del tiempo y se habían convertido en figuras en buena medida impersonales. Esta impersonalidad era una característica definitoria de la auctoritas $^{30}$. De allí el carácter en buena me-

\footnotetext{
${ }^{28}$ Conv. I.i.18: "E con ciò sia cosa che la vera intenzione mia fosse altra che quella che di fuori mostrano le canzoni predette, per allegorica esposizione quelle intendo mostrare, appresso la litterale istoria ragionata; sì che l'una ragione e l'altra darà sapore a coloro che a questa cena sono convitati".

${ }^{29}$ Conv. I.ix.10: "Ancora, darà lo volgare dono non dimandato, che non l'averebbe dato lo latino: però che darà se medesimo per comento, che mai non fu domandato da persona; e questo non si può dire de lo latino, che per comento e per chiose a molte scritture è già stato domandato, sì come ne'loro principii si può vedere apertamente in molte. E così è manifesto che pronta liberalitade mi mosse al volgare anzi che a lo latino".

${ }^{30}$ Cf. A. Russell Ascoli, (2005, p. 46): "Remarkable as such an assertion [la de Dante] of individual creative authorship might seem in any time and place, it seems far more so in late thirteenth- to early fourteenth-century Italy, a time when authority in all areas (political, theological, philosophical, even literary) was conceived of as fundamentally impersonal. Originating in a timeless transcendent truth, the empirical evidence for auctoritas was antiquity (the great classical Latin texts which had survived the test of time were known as auctores), genealogical line-
} 
dida paradójico del proyecto dantesco de construir una autoridad personal: la autoridad de Dante. De allí también la aparición insistente de ese "yo" sobre el cual reposa la argumentación y que, a su vez, es autorizado por esa misma argumentación que él construye, justamente, con la finalidad de autorizarse. Esto se pone de manifiesto en un pasaje que muestra la equivalencia que Dante establece entre la situación de exilio que afecta a su persona, y que lo ha conducido a presentarse físicamente ante hombres que desconocían su obra, lo cual le ha restado valor y autoridad, y la situación de esa misma obra, también ella minusvalorada. El Convivio viene a reparar esta situación de exilio que viven tanto la obra cuanto su autor:

Por eso, dado que, como se ha dicho más arriba, yo me he presentado a casi todos los italianos, y por este hecho me he mostrado quizás más vil de lo que quiere la verdad, no sólo frente a aquellos a quienes ya había llegado mi fama, sino también frente a los otros, para quienes sin duda mis cosas -y yo mismohan disminuido su valor; [por eso, digo,] me es preciso dar, con un más alto estilo, en esta obra, un poco más de gravedad, gracias a la cual aparezca de mayor autoridad (subr. prop.) ${ }^{31}$.

Aquí aparece la tríada conceptual "verdad / estilo / autoridad", tríada clave en la comprensión de las motivaciones de esta obra. Los vínculos entre estos conceptos son significativos. Dante afirma que el exilio lo obligó a presentarse fisicamente ante sus connacionales, los itálicos. La presencia física ${ }^{32}$ de su persona lo ha conducido a una situación en la que se impone la falsedad: su obra y su persona han disminuido su valor ("le mie cose sanza dubbio meco sono alleviate") de un modo que no conviene al ser real de esas cosas (la vida y la obra).

La analogía entre el texto y la persona (letra / cuerpo, sentido / alma) opera como el vínculo entre la situación del autor exiliado y la situación de su obra, cuyo "exilio" consiste en verse reducida a su aspecto exclusivamente material, literal, en suma, a su belleza. El Convivio viene a remediar esa situación de falsedad producida por un exilio a la vez biográfico (en lo que hace a la persona) y alegórico (en lo que hace a la obra). Mediante un estilo más alto ("con più alto stilo"), el Convivio debe dar a su autor una mayor dignidad, una imagen de seriedad (tales son los sentidos de "gravezza") que lleve al autor y a su obra a conseguir la autoridad de la cual se ha visto privado por las consecuencias del

\footnotetext{
age (aristocratic families), or the hierarchical rights conferred by office (notably the papacy and the imperial throne, both obtained by election). Dante, especially after his expulsion from Florence, had none of the attributes that could transform his personal ambitions and visions into an impersonal auctoritas: whith claims to only the most minor of aristocratic origins (see Paradiso 15-17), he lacked the standing conferred by public office, had none of the rights and privileges associated with even minor ecclesiastical orders, and possessed no significant wealth with which he could purchase the titles that he lacked".

${ }^{31}$ Conv. I.iv.13: "Onde con ciò sia cosa che, come detto è di sopra, io mi sia quasi a tutti li Italici appresentato, per che fatto mi sono più vile forse che 'l vero non vuole non solamente a quelli a li quali mia fama era già corsa, ma eziandio a li altri, onde le mie cose sanza dubbio meco sono alleviate; convienmi che con più alto stilo dea, ne la presente opera, un poco di gravezza, per la quale paia di maggiore autoritade". Nótese la oposición liviano/grave (alleviare/gravezza) vinculada a la autoridad (Stabile, 1984).

${ }^{32}$ Una presencia literal, pues el cuerpo aparece como la letra del alma, así como la letra es el cuerpo del sentido: el aspecto exterior en que se muestra el sentido más profundo y verdadero.
} 
exilio y de la mala comprensión de los poemas por parte de aquellos lectores que sólo atendían a su belleza.

La situación de Dante es, pues, análoga a la de su obra. Los italici sólo perciben de ambos los rasgos exteriores, el aspecto físico del cuerpo y de las letras; por más que se muestren reales, esas apariencias son falsas. Por eso, la operación hermenéutica del Convivio consiste en romper el hechizo físico de la literalidad, que muestra a la cosa en su puro aparecer, es decir, en su falsedad. Esta reinterpretación alegórica de los poemas se traslada se traslada también al autor / auctor, esto es, al hombre. De este modo, la hermenéutica alegórica hace aparecer en la cosa -el hombre y sus canciones- un sentido que parece ser ajeno (alienum) a la cosa misma y que, sin embargo, es la verdad de la cosa ${ }^{33}$.

\section{El Convivio actualiza la esencia filosófica de la lengua vernácula}

Dante afirma haber optado por el uso del vulgar para su comentario en virtud del "amor natural" ("naturale amore") que siente por su propia lengua ${ }^{34}$. El amor natural impulsa al amante a tres cosas: a magnificar al amado, a celarlo y a defenderlo. Estos tres motivos inclinaron a Dante a adoptar su lengua materna en el comentario de sus canciones ${ }^{35}$. Aquí nos interesan especialmente el primero y el último de estos motivos, esto es, la magnificación y la defensa del amado por el amante.

La operación de escribir el comentario fílosófico de sus canciones en lengua vernácula conduce a magnificar esta misma lengua, pues pone de manifiesto una "bondad" que antes de realizada esa operación permanecía oculta:

Me impulsó primeramente el [deseo de] magnificarlo. Y que en este [comentario] yo lo magnifico, se puede ver por esta razón; dado que por muchas condiciones de grandeza se pueden magnificar las cosas, esto es, hacerlas grandes, y ninguna vuelve tan grande [a la cosa magnificada] cuanto la grandeza de la propia bondad, que es madre y conservadora de todas las otras grandezas; por eso el hombre no puede tener ninguna grandeza mayor que aquella de la operación virtuosa, que es su propia bondad, por la cual son conservadas y adquiridas las grandezas de las verdaderas dignidades, de los verdaderos honores, de las verdaderas potencias, de las verdaderas riquezas, de los verdaderos amigos, de la verdadera y clara fama: y yo doy esta grandeza a este amigo, en cuanto, aquello que él tenía de bondad en potencia y oculto, yo se lo hago tener en acto y abiertamente en su propia operación, que es manifestar la doctrina concebida $^{36}$.

\footnotetext{
${ }^{33}$ Cf. Dante Alighieri, Ep. XIII, 7: "Nam allegoria dicitur ab alleon graece, quod in latinum dicitur alienum, sive diversum".

${ }^{34}$ Dante encuentra tres razones que lo excusan por el uso del vulgar: 1) "la precaución de evitar un desorden no conveniente"; 2) "mi deseo de ejercer pronto mi liberalidad"; 3 ) "el natural amor al propio lenguaje", es decir: orden, virtud (liberalidad) y amor.

${ }^{35}$ Cf. Conv. I.x.6: "Dico che lo naturale amore principalmente muove l'amatore a tre cose: l'una si è a magnificare l'amato; l'altra è a essere geloso di quello; l'altra è a difendere lui, sì come ciascuno può vedere continuamente avenire. E queste tre cose mi fecero prendere lui, cioè lo nostro volgare, lo quale naturalmente e accidentalmente amo ed ho amato".

${ }^{36}$ Conv. I.x.7-9: "Mossimi prima per magnificare lui. E che in ciò io lo magnifico, per questa ragione vedere si può: avegna che per molte condizioni di grandezze le cose si possano magnificare, cioè fare grandi, nulla fa tanto grande quanto la grandezza della propia bontade, la quale è madre e conservatrice dell'altre grandezze. Onde nulla grandezza puote l'uomo avere maggiore che quella della virtuosa operazione, che è sua propia bontade; per la quale le grandezze delle vere dignitadi, delli veri onori, delle vere potenze, delle vere ricchezze, delli veri amici, della ve-
} 
La conclusión es sorprendente. Al escribir, por primera vez, un comentario en lengua vulgar, Dante actualiza la esencia filosófica de esta lengua vernácula, esto es, la capacidad del vulgar de expresar conceptos ("manifestare conceputa sentenza"). La lengua vernácula posee una potencialidad que hasta el momento permanecía inactualizada. Había una "bondad" propia de esa lengua que no había sido aún desarrollada. Este es el nuevo paso que Dante afirma estar dando en el Convivio: mostrar una capacidad (potentia, dýnamis, se trata evidentemente de la teoría aristotélica de la potencia y el acto) del lenguaje vulgar que, hasta él, había permanecido oculta. Este pasaje de la potencialidad al acto (de lo que está "in podere e occulto" a lo que está "in atto e palese") implica, aristotélicamente, un perfeccionamiento de la cosa actualizada (Segre, 1963). Pero esta capacidad que la prosa de Dante actualiza no es una potencialidad accidental: se trata de la esencia misma, es decir, de la operación propia del agente ("sua propia operazione"). Esta operación propia -el fin al que la cosa tiendeconsiste en expresar la doctrina ("sentenza"). De modo que la propria operatio del vulgar -como de toda lengua- es de orden filosófico ("manifestare conceputa sentenza"), y filosófica es también, en consecuencia, su finalidad. La filosofía parece estar inscripta en la finalidad propia de la lengua:

“Así el lenguaje, que está ordenado a manifestar el concepto humano, es virtuoso cuando lo hace, y es más virtuoso aquel que lo hace mejor" (Conv. I.v.12). "Y nosotros vemos que en todo lo relativo al lenguaje, lo más amado y encomiado es el manifestar bien el concepto: por lo tanto, esta es su primera bondad" (Conv. I.xii.13). "Si consideramos atentamente qué es lo que hacemos cuando hablamos, aparece que no es nada más que comunicar a los otros lo que nuestra mente ha pensado" (De vulg. eloq. I.ii.3) ${ }^{37}$.

El Convivio tiene un alcance mucho mayor que el de ser una mera divulgación enciclopédica. En él, Dante otorga por primera vez a la lengua materna la grandeza ("e questa grandezza do io a questo amico") que consiste en desarrollar su virtud propia, esto es, manifestar conceptos. Sin su intervención, la potencialidad de la lengua vernácula no habría pasado al acto, y la naturaleza habría obrado en vano. Por este motivo, Roger Dragonetti ha señalado que Dante se propone revelar en la palabra el ser del lenguaje ${ }^{38}$, actualizar su esencia filosófica: "e questa grandezza do io a questo amico, in quanto quello che elli di bontade avea in podere e occulto, io lo fo avere in atto e palese ne la sua propria operazione, che è manifestare la conceputa sentenza". Sacar de la oscuridad, llevar a la luz, cumplir el tránsito de la potencia al acto, en suma, hacer que la esencia del lenguaje se explicite, he aquí la función que Dante afirma estar llevando a cabo.

\footnotetext{
ra e chiara fama e acquistate e conservate sono. E questa grandezza do io a questo amico, in quanto quello che elli di bontade avea in podere e occulto, io lo fo avere in atto e palese nella sua propia operazione, che è manifestare conceputa sentenza".

${ }^{37}$ Cf. Conv. I.v.12: "Così lo sermone, lo quale è ordinato a manifestare lo concetto umano, è virtuoso quando quello fa, e più virtuoso quello che più lo fa". También I.xii.13: "E noi vedemo che in ciascuna cosa di sermone lo bene manifestare del concetto è più amato e commendato: dunque è questa la prima sua bontade". Cf. De vulg. eloq. I.ii.3: "Si etenim perspicaciter consideramus quid cum loquimur intendamus, patet quod nichil aliud quam nostre mentis enucleare aliis conceptum".

${ }^{38}$ Cf. R. Dragonetti (2006, p. 47): "La révélation de l'être du langage dans la parole est le plus grand don que le poète reconnaissant puisse offrir au langage lui-même, et c'est là le don que Dante apporte à sa langue maternelle dans le Convivio (I.x.9)".
} 
Se comprende, entonces, la radicalidad del proyecto filosófico del Convivio. Este proyecto consiste en perfeccionar la naturaleza de la lengua vernácula mediante la actualización de sus potencialidades $\mathrm{y}$, de este modo, hacer que la entidad "lengua vernácula" cumpla la finalidad que le es propia. Esta finalidad consiste en la transmisión de lo concebido por el hombre (dottrina, sentenza, concetto $)^{39}$.

Dante continúa el mismo argumento al desarrollar el último de los motivos alegados en la elección del vulgar: la defensa del amado. Una vez más, el argumento es fundamentalmente metafísico (se trata de realizar el tránsito de la potencia al acto y manifestar así la virtud del vulgar), esto es, a partir de la explicitación del ser de la lengua vulgar Dante muestra en qué consisten su virtud o bondad propias. Vemos reaparecer, en este nuevo argumento, la virtud filosófico-conceptual de la lengua vernácula:

Me impulsó, aún, el [deseo de] defender al vulgar de muchos de sus acusadores, que lo desprecian a él y encomian a los otros vulgares, principalmente al de la lengua de "oc", diciendo que este último es más bello y mejor que aquel; y en esto se alejan de la verdad. Por medio de este comentario se verá la gran bondad del vulgar del "sì" [el italiano]; puesto que se verá su virtud, tal como la de manifestar, mediante él, altísimos y novísimos conceptos de un modo conveniente, suficiente y adecuado, casi del mismo modo que en el latín ${ }^{40}$.

Al desplegarse en un comentario filosófico, la lengua vernácula se muestra casi a la altura del latín. Dante afirma -y el Convivio debe ser una prueba de esta afirmación- que la virtud del vulgar consiste en que también él puede expresar "altísimos y novísimos conceptos" ("altissimi e novissimi conce$t t i$ '). La novedad de los conceptos consiste en que, hasta este proyecto dantesco, la lengua vernácula no había sido utilizada filosóficamente: "El vulgar dará un regalo no solicitado, pues se dará a sí mismo como comentario, no habiendo sido jamás solicitado por ninguna persona"41. Así, el fin metafísico coincide aristotélicamente- con la virtud propia de la cosa, es decir, con el cumplimiento de su fin ético ${ }^{42}$. El Convivio entero ha de ser una demostración de la conve-

\footnotetext{
${ }^{39}$ Por eso Dragonetti (2006, p. 47), al comentar este mismo pasaje (Conv. I.x.7-9), sostiene que "Dante est le véritable trouveur de sa langue maternelle, puisque, par amour, il l'a révélée à ellemême dans son être. C'est pour quoi le Convivio n'est pas seulement la solution d'un conflit intérieur entre deux modes de vie poétique, mais une aurore nouvelle, puisqu'il s'agit de célébrer une naissance insigne: l'avènement à la parole, de l'être du langage vulgaire".

${ }^{40}$ Conv. I.x.11-12: "Mossimi ancora per difendere lui da molti suoi accusatori, li quali dispregiano esso e commendano li altri, massimamente quello di lingua d'oco, dicendo che è più bello e migliore quello che questo; partendose in ciò da la veritade. Chè per questo comento la gran bontade del volgare di sì si vedrà; però che si vedrà la sua vertù, sì com'è per esso altissimi e novissimi concetti convenevolemente, sufficientemente e acconciamente, quasi come per esso latino, manifestare".

${ }^{41}$ Conv. I.ix.10: "Darà lo volgare dono non dimandato, che non l'averebbe dato lo latino: però che darà se medesimo per comento, che mai non fu domandato da persona".

${ }^{42}$ Todo este pasaje tiene un fuerte contenido aristotélico. Además del evidente parentezco conceptual, Dante cita explícitamente una traducción de la Ética de Aristóteles cuando afirma que eligió escribir su comentario en vulgar por el celo que se tiene al amado: "Mossimi secondamente per gelosia di lui. La gelosia dell'amico fa l'uomo sollicito a lunga provedenza. Onde, pensando che lo desiderio d'intendere queste canzoni a alcuno illitterato averebbe fatto lo comento latino transmutare in volgare, e temendo che '1 volgare non fosse stato posto per alcuno che l'avesse laido fatto parere, come fece quelli che transmutò lo latino dell'Etica - ciò fue Taddeo ipocratista -, providi a ponere lui, fidandomi di me più che d'un altro" (Conv. I.x10).
} 
niencia, suficiencia y adecuación de la lengua italiana a la expresión filosófica y conceptual ("per esso [volgare] altissimi e novissimi concetti convenevolemente, sufficientemente e acconciamente, quasi come per esso latino, manifestare").

\section{La traducción de la poesía a la filosofía}

De la Vita nuova al Convivio se produce el tránsito de la poesía a la filosofía, y, al interior del Convivio mismo, se opera una traducción del verso a la prosa conceptual. Dante toma consciencia de que la potencia (vertù) filosófica de la lengua vernácula sólo podía ser puesta de manifiesto (actualizada) no ya mediante la estructura rítmica del verso, sino gracias al desarrollo de una prosa argumentativa:

Esa virtud [de la lengua vulgar] no se podía manifestar en las cosas rimadas, a causa de los adornos accidentales que aquí están estrechamente unidos, esto es, la rima y el ritmo y el número regulado: tal como no se puede manifestar adecuadamente la belleza de una dama cuando los adornos del tocado y de la vestimenta se vuelven más admirables que ella misma. Por eso, quien quiere juzgar adecuadamente a una dama, mírela cuando sólo tenga con ella su belleza natural y no esté acompañada por ningún adorno accidental: tal como será este comentario [italiano], en el cual se verá la facilidad de sus sílabas, las propiedades de sus construcciones y las suaves oraciones que se hacen de él: las cuales quien observe bien verá llenas de dulcísima y amabilísima belleza ${ }^{43}$.

Por contraposición a la poesía, que aparece aquí como una dama afeitada en exceso, y, por lo tanto, artificiosa, la filosofía aparece como una dama de belleza natural, como una dama desnuda. Frente a la artificiosidad de las rimas, el Convivio ha de poner en obra el curso natural de la prosa itálica. Estos artificios poéticos, lejos de haber mostrado la virtud de la lengua, la han ocultado, como el exceso de vestido oculta la belleza de una dama.

Dante no opone aquí la belleza a la verdad. La oposición se produce entre dos tipos de belleza: la belleza en cierto modo excesiva y accidental (" $a c$ cidentale adornamento") de la poesía frente a la belleza natural de la prosa filosófica ("questo comento"). La belleza poética ponía de manifiesto la rima, el ritmo y el metro de la lengua, es decir, la melodía del lenguaje; la belleza filosófica pone ahora de manifiesto la facilidad de las sílabas, los períodos sintácticos y la suavidad de la lengua, es decir, su capacidad de persuasión ${ }^{44}$, y todo esto hará aparecer la dulzura y la belleza propia de la lengua italiana.

\footnotetext{
${ }^{43}$ Conv. I.x.12-13: "La quale [vertù] non si potea bene manifestare ne le cose rimate, per le accidentali adornezze che quivi sono connesse, cioè la rima e lo ritimo e lo numero regolato: sì come non si può bene manifestare la bellezza d'una donna, quando li adornamenti de l'azzimare e de le vestimenta la fanno più ammirare che essa medesima. Onde chi vuole ben giudicare d'una donna, guardi quella quando solo la sua naturale bellezza si sta con lei, da tutto accidentale adornamento discompagnata: sì come sarà questo comento, nel quale si vedrà l'agevolezza de le sue sillabe, le proprietadi de le sue costruzioni e le soavi orazioni che di lui si fanno; le quali che bene agguarderà, vedrà piene di dolcissima e d'amabilissima bellezza".

${ }^{44}$ La suavitas (suavidad de la elocución) es condición del suadere (persuadir). El complejo semántico suavidad, persuasión y dulzura aparece con claridad en Conv. II.vii.5: "Dico adunque che vita del mio core, cioè del mio dentro, suole essere un pensiero soave ('soave' è tanto quanto 'suaso', cioè abbellito, dolce, piacente e dilettoso)". Cf. R. Dragonetti, 2006, pp. 229-256.
} 
Dante ha afirmado que la virtud del lenguaje ("sermone") consiste en "manifestar el concepto humano" ("manifestare lo concetto umano"), y que una lengua es más virtuosa cuanto mejor lo manifiesta. Aunque la virtud del latín sea superior a la del vulgar, pues puede manifestar "muchas cosas concebidas en la mente que el vulgar no puede manifestar" $"$, de todos modos el vulgar posee también esta "bondad" (la capacidad de expresar conceptos), aunque todavía esta virtud no sea perfecta, ya que no existe la costumbre de utilizar la lengua vernácula filosóficamente ${ }^{46}$. La prosa filosófica del Convivio contribuirá a que el vulgar adquiera ese hábito que le falta para acceder al mismo status que el latín.

Esta expresión de la verdad conceptual en el uso filosófico de la lengua no es enemiga del uso poético del lenguaje. Ambas son complementarias, como muestra el caso del latín. La belleza consiste en una armonía - una correspondencia entre las partes- de la cual resulta placer ("della loro armonia resulta piacimento"). El latín es más bello que el vulgar porque la correspondencia entre sus partes -es decir, la estructura sintáctica de la oración o la composición métrica del verso- sigue el arte, mientras que el vulgar sólo se guía por el uso ${ }^{47}$. Pero esta imperfección del vulgar no es absoluta, esto es, no es una imperfección del ser de la lengua vernácula, sino que es relativa a su evolución. En cuanto a belleza, es decir, en lo que hace a la correspondencia métrica de sus partes, el vulgar ha alcanzado ya una cierta madurez, de la cual Dante mismo es en parte responsable, pues él ha contribuido a ligar "con número y rimas" las palabras de esa lengua. Al hacer esto, Dante ha contribuido a la conservación de la lengua italiana:

Toda cosa tiende (studia) naturalmente a su conservación: por lo cual, si el vulgar pudiese desear (studiare) por sí mismo, tendería (studierebbe) a aquella; y aquella consistiría en prepararse a sí mismo para tener mayor estabilidad, y no podría tener mayor estabilidad que en ligarse con número y con rimas. Y esto mismo ha sido mi deseo (studio), lo cual es tan evidente que no requiere testimonio. Por lo cual un mismo deseo (studio) ha sido el suyo y el mío; por eso la amistad se ha confirmado y ha crecido a partir de esta concordia ${ }^{48}$.

\footnotetext{
${ }^{45}$ Cf. Conv. I.v.12: "Così lo sermone, lo quale è ordinato a manifestare lo concetto umano, è virtuoso quando quello fa, e più virtuoso quello che più lo fa; onde, con ciò sia cosa che lo latino molte cose manifesta concepute nella mente, che lo volgare fare non può, sì come sanno quelli che hanno l'uno e l'altro sermone, più è la vertù sua che quella del volgare".

${ }^{46}$ Cf. Conv. I.xii.12-13: "Di questa vertù inanzi dicerò più pienamente nel quartodecimo trattato; e qui lasciando, torno al proposito. Provato è adunque la bontà della cosa più propia più essere amabile in quella. E a mostrare quale in essa è più propia, è da vedere quella che più in essa è amata e commendata, e quella è essa. E noi vedemo che in ciascuna cosa di sermone lo bene manifestare del concetto è più amato e commendato: dunque è questa la prima sua bontade. $\mathrm{E}$ con ciò sia cosa che questa sia nel nostro volgare, sì come manifestato è di sopra in altro capitolo, manifesto è che ella è delle cagioni stata dell'amore ch'io porto ad esso: poi che, sì come detto è, la bontade è cagione d'amore generativa".

${ }^{47}$ Cf. Conv. I.v.13-14: "Quella cosa dice l'uomo essere bella, cui le parti debitamente si rispondono, per che della loro armonia resulta piacimento. Onde pare l'uomo essere bello, quando le sue membra debitamente si rispondono; e dicemo bello lo canto, quando le voci di quello, secondo 'l debito dell'arte, sono intra sé rispondenti. Dunque quello sermone è più bello, nello quale più debitamente si rispondono li vocabuli; e più debitamente li vocabuli si rispondono in latino che in volgare, però che lo volgare séguita uso, e lo latino arte. Onde concedesi esser più bello, più virtuoso e più nobile".

${ }^{48}$ Conv. I.xiii.6-7: "Ciascuna cosa studia naturalmente alla sua conservazione: onde, se lo volgare per sé studiare potesse, studierebbe a quella; e quella sarebbe aconciare sé a più stabilitate, e più stabilitate non potrebbe avere che in legar sé con numero e con rime. E questo medesimo
} 
En el proyecto filosófico que Dante comienza en el Convivio no se trata ya de conservar la lengua materna mediante su armonización, es decir, mediante la actualización de su belleza a través de los vínculos métricos, rítmicos y sintácticos que establece la poesía, sino de contribuir a actualizar la virtud propia de toda lengua y, en consecuencia, también de la lengua vulgar: desplegar sus propias potencialidades filosóficas.

\section{La intraducibilidad del poema}

Verdad y belleza, hemos señalado, no se oponen en el pensamiento de Dante: se complementan. Por eso la prosa filosófica del Convivio ha de ser accesible a los mismos hombres que sean capaces de entender las canciones comentadas: la verdad sin la experiencia de la belleza, o la belleza sin el conocimiento de la verdad, aparecen como insuficientes a los ojos de Dante. Este es otro de los motivos de la elección del vulgar. En efecto, si el comentario a las canciones hubiese sido escrito en latín, habría podido ser leído por extranjeros, quienes habrían llegado comprender el comentario sin entender plenamente las canciones mismas, ya que al desconocer la lengua original no podrían haber experimentado la belleza del lenguaje, y sólo habrían conocido su sentido en traducción. La belleza de la lengua en su aspecto sonoro (metro, rima, ritmo) es característica esencial de la poesía. Para comprender la verdad que permanece oculta bajo el entramado de rimas, ritmo y métrica es necesario poder atravesar ese entramado en lo que tiene de significativo, aunque ese significado del verso sea solamente una parte del significado total del poema. El poema contiene (porta) y transmite una verdad que lo excede. Pero, a su vez, esa verdad sólo puede ser transmitida y contenida por el poema:

El latín habría expuesto [las canciones] a gente de otra lengua, como a los alemanes, a los ingleses y a otros, y aquí habría sobrepasado la orden recibida [por su señor, es decir, las canciones,] porque, contra su voluntad, digo, hablando en general, habría sido expuesto su significado alli donde ellas no lo podían llevar con su belleza. Y por eso sépase que ninguna cosa armonizada por los lazos de las musas se puede traducir a otra lengua sin romper toda su armonía y su dulzura. Y esta es la razón por la que Homero no se tradujo del griego al latín como los otros textos que tenemos de ellos. Y esta es la razón por la que los versos del Salterio carecen de música y de armonía; pues fueron traducidos del hebreo al griego y del griego al latín, y en la primera traducción toda aquella dulzura desapareció" (subr. prop.) ${ }^{49}$.

\footnotetext{
studio è stato mio, sì come tanto è palese che non dimanda testimonianza. Per che uno medesimo studio è stato lo suo e 'l mio: per che di questa concordia l'amistà è confermata e acresciuta".

${ }^{49}$ Conv. I.vii.13-15: "Anche: lo latino l'averebbe esposte a gente d'altra lingua, sì come a Tedeschi e Inghilesi e altri, e qui averebbe passato lo loro comandamento; ché contra loro volere, largo parlando dico, sarebbe essere esposta la loro sentenza colà dov'elle non la potessero colla loro bellezza portare. E però sappia ciascuno che nulla cosa per legame musaico armonizzata si può della sua loquela in altra transmutare sanza rompere tutta sua dolcezza ed armonia. E questa è la cagione per che Omero non si mutò di greco in latino, come l'altre scritture che avemo da loro. E questa è la cagione per che i versi del Salterio sono sanza dolcezza di musica e d'armonia: ché essi furono transmutati d'ebreo in greco e di greco in latino, e nella prima transmutazione tutta quella dolcezza venne meno".
} 
La belleza de las canciones aparece como una condición necesaria de la transmisibilidad del sentido, pues el sentido de las canciones está involucrado en su belleza. Por eso, al traducir los poemas se pierde la verdad propiamente poética, y no queda más que un texto en prosa que carece tanto de la dulzura y de la armonía propias del verso cuanto de la dulzura propia de la prosa ${ }^{50}$.

Al comentar el sentido literal de la tornada de Voi che 'ntendendo il terzo ciel movete, Dante vuelve sobre la distinción entre belleza y verdad (sentido, bondad) ${ }^{51}$, señalando esta vez el placer (“diletto") que ambas producen:

La bondad y la belleza de todo discurso son diversas entre sí y están separadas; pues la bondad se encuentra en el sentido y la belleza se encuentra en el ornato de las palabras; una y otra producen deleite, aunque la bondad sea máximamente deleitable ${ }^{52}$.

La dificultad -a la vez que el límite- de la expresión poética consiste en que es incapaz de expresar la "bondad" del discurso, es decir, su sentido, pues la belleza, como el exceso de maquillaje, la cubre con sus adornos. En consecuencia, el goce ("diletto") producido por la poesía es menor que el producido por la prosa filosófica, pues es incompleto. Por este motivo los poemas tienen necesidad de un comentario. Dante expone este motivo al desarrollar el sentido literal de la tornada:

Digo pues: Yo creo, canción, que escasos son, es decir, pocos, los que te entienden bien. Y digo la causa, que es doble. Primera: porque hablas fatigosamente -digo "fatigosamente" por la causa que se ha señalado-; luego: porque hablas de un modo duro - digo "duro" en cuanto a la novedad de su sentido-. Luego la amonesto y digo: Si por ventura sucede que tú vas allí donde haya personas que duden de tus razonamientos, no te pierdas, antes bien diles: Dado que no veis mi bondad, parad mientes al menos en mi belleza. Con lo cual, como se ha dicho más arriba, no quiero decir sino esto: Ah, hombres, que no podéis ver el sentido de esta canción, no la refutéis sin embargo; parad mientes en su belleza, que es grande tanto por su construcción, que compete a los gramáticos, cuanto por el orden del discurso, que compete a los retóricos, y por el número de sus partes, que compete a los músicos. Estas cosas se pueden ver fácilmente en ella, para quien bien mira ${ }^{53}$.

\footnotetext{
${ }^{50}$ P. Toynbee, op. cit., pp. 51-52 ha señalado como fuente del pasaje dantesco la carta prefacio de San Jerónimo a su traducción de la Crónica de Eusebio (Interpretatio Chronicae Eusebii, PL, XXVII, 34). En su edición del Convivio, Busnelli-Vandelli (ad locum) señalan que la intertextualidad fue descubierta, antes que por Toynbee, por Scherillo, a partir de la carta Var. 25 de Petrarca, y luego por Marigo. Dante Alighieri, Il convivio, ridotto a miglior lezione e commentato da G. Busnelli e G. Vandelli, Le Monnier, Firenze, Vol. I: 1934, Vol. II: 1937.

${ }^{51}$ Los tres últimos términos pertenecen al mismo campo semántico -que alude a la esencia de la cosa-, pero refieren a tres aspectos diferentes: la verdad refiere al aspecto ontológico, el sentido al semántico y la bondad al ético, aunque este último también pueda aludir a la bondad de la cosa en tanto que su fin, es decir, al aspecto metafísico.

${ }^{52}$ Conv. II.xi.4: "La bontade e la bellezza di ciascuno sermone sono intra loro partite e diverse; chè la bontade è ne la sua sentenza, e la bellezza è ne l'ornamente de le parole; e l'una e l'altra è con diletto, avvegna che la bontade sia massimamente dilettosa".

${ }^{53}$ Conv. II.xi.7-9: "Dico adunque: Io credo, canzone, che radi sono, cioè pochi, quelli che intendano te bene. E dico la cagione, la quale è doppia. Prima: però che faticosa parli -'faticosa' dico per la cagione che detta è-; poi: però che forte parli -'forte' dico quanto a la novitade de la sentenza-. Ora appresso ammonisco lei e dico: Se per avventura incontra che tu vadi là dove persone siano che dubitare ti paiano ne la tua ragione, non ti smarrire, ma dì loro: Poi che non vedete la mia bontade, ponete mente almeno a la mia belleza. Che non voglio in ciò altro dire, secondo ch'è detto di sopra, se non: O uomini, che vedere non potete la sentenza di questa canzone, non
} 
Este comentario dantesco reproduce, casi mot-à-mot, la tornada de la canción, y echa nueva luz sobre el vínculo que establecen belleza y verdad en el pensamiento de Dante en el Convivio. Dante pareciera sugerir que la belleza, dado que contiene (porta) en sí misma la verdad, logra transmitir algo de ella aun a aquellos que son incapaces de comprenderla. Esta transmisión parece darse de un modo "inconsciente": quienes sólo perciben la belleza no son conscientes de que allí mismo reside, oculto, el sentido, la bondad del poema:

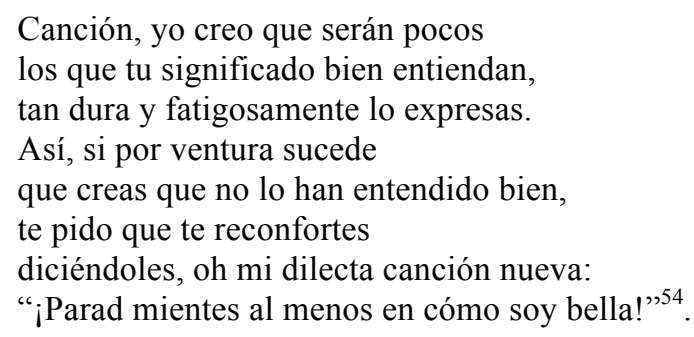

Como en el Banquete platónico, en el Convivio dantesco la belleza -y muy especialmente la belleza del poema- es una puerta de acceso al bien y a la verdad. Este vínculo que establecen la belleza y la verdad, y que posibilita el tránsito de la poesía a la filosofía, está a la base del proyecto dantesco de escribir filosofía en lengua vernácula. Este proyecto tiene, a la vez como condición de posibilidad y como límite, un proceso de traducción que garantice el tránsito de la belleza poética a la prosa filosófica, esto es, un proceso de traducción gracias al cual se explicite la virtud filosófico-conceptual que yacía en potencia y oculta en los versos. La filosofía tiene el cometido de explicitar esa verdad que la poesía presenta oculta bajo la belleza. Poesía, filosofía y traducción se encuentran así inescindiblemente unidas en el proyecto del Convivio.

\author{
Mariano Pérez Carrasco \\ mperezcarrasco78@yahoo.com \\ Prof. doutor, Universidad de Buenos Aires
}

\footnotetext{
la rifiutate però; ma ponente mente la sua bellezza, ch'è grande sì per costruzione, la quale si pertiene a li gramatici, sì per l'ordine del sermone, che si pertiene a li rettorici, sì per lo numero de le sue parti, che si pertiene a li musici. Le quali cose in essa si possono belle vedere, per che ben guarda".

${ }^{54}$ Canzone prima, vv. 53-61: "Canzone, io credo che saranno radi / color che tua ragione intendan bene, / tanto parli faticosa e forte. / Onde, se per ventura alli addivene / che tu dinanzi da parsone vadi / che non ti paian d-essa bene accorte, / allor ti priego che ti riconforte, / dicendo lor, diletta mia novella: "Ponete mente almen com'io son bella!",".
} 


\section{Bibliografía}

Alighieri, Dante. Convivio. F. Brambilla Ageno (a cura di). Edizione Nazionale / Le Letttere: Firenze, 1995. Disponible en formato electrónico: http://etcweb.princeton.edu/dante/pdp/convivio.html . Monarchia. P. G. Ricci (a cura di). Mondadori: Milano, 1965.

. De vulgari eloquentia. Vicenzo Coletti (a cura di). Garzanti: Torino, 1995.

. Commedia. A. M. Chiavacci Leonardi (a cura di). Mondadori: Milano, 2009.

. Rime. Gianfranco Contini (a cura di). Einaudi: Milano, 1980.

Bibliografía secundaria

BeRTELloni, Francisco. Introducción: La vida y el periplo intelectual de Dante. In: Dante Alighieri. Convivio. Traducción de M. Pérez Carrasco. Colihue: Buenos Aires, 2008.

BiAnchI, Luca. Il vescovo e i filosofi. La condanna parigina del 1277 e l'evoluzione dell'aristotelismo scolastico. Pierluigi Lubrina: Bergamo, 1990.

. Filosofi, Uomini e Bruti. Note per la Storia di un'Antropologia 'Averroista'. In: As Relações de poder no pensamento político da Baixa Idade Média. Homenagem a J. M. Barbosa. Revista da Faculdade de Ciências Sociais e Humanas, No. 7, Vol. I: Lisboa, 1994, pp. 105-126.

De LiBera, Alain. Penser au Moyen Âge. Seuil: Paris, 1991.

De Lubac, Henri. Exégèse Médiévale. Les quatre sens de l'Écriture, T. III, Seconde Partie, I. Aubier: Paris, 1961.

DragonetTI, Roger. La conception du langage poétique dans le De vulgari eloquentia. In: R. Dragonetti. Dante, la langue et le poème. Belin: Gèneve, 2006, pp. 45-106.

. Le style suave dans le Convivio et la Divina Commedia. In: R. Dragonetti. Dante, la langue et le poème. Belin: Gèneve, 2006, pp. 229-256.

GILson, Etienne. Dante et la philosophie. Vrin: Paris, 1937.

Hollander, Robert. Allegory in Dante's Commedia. Princeton University Press: Princeton, 1969.

KANTOROWICZ, Ernst. Los dos cuerpos del rey. Un estudiode teología política medieval. Traducción de S. Aikin Araluce y R. Blázquez Godoy. Alianza: Madrid, 1985.

LE Goff, Jacques. Los intelectuales en la Edad Media. Traducción de M. A. Galmarini, con revisión técnica de N. Guglielmi. Eudeba: Buenos Aires, 1965.

MARTI, Mario. Storia dello Stil Nuovo. Milella: Lecce, 1974.

NietzSCHE, Friedrich. Genealogía de la moral. Traducción de A. Sánchez Pascual. Alianza: Madrid, 1997.

Ortega y Gasset, José. Apuntes sobre el pensamiento. Su teurgia y su demiurgia. Revista de Occidente: Madrid, 1959.

PEPIN, Jean. Dante et la tradition de l'allegorie. Institut d'Études MédiévalesVrin: Montreal-Paris, 1971. 
RAGGIUNTI, R. Introduzione a Husserl. Laterza: Bari, 1986.

Russell AsColi, Albert. Dante and the Making of a Modern Author. Cambridge University Press: Cambridge, 2005.

Segre, Cesare. Il Convivio di Dante Alighieri. In: C. Segre. Lingua, stile e società. Studi sulla storia della prosa italiana. Feltrinelli: Milano, 1963, pp. 227-228.

Singleton, Charles. Dante's Allegory. In: Speculum, Vol. 25, No. 1. Medieval Accademy of America, 1958, pp 78-86.

Stabile, Giorgio. Autorità, autoritade. In: Enciclopedia Dantesca. Istituto della Enciclopedia Italiana: Roma, 1984, Vol. I, pp. 456-460.

TeEuwen. The Vocabulary of Intellectual Life in the Middle Ages. Comité International du Vocabulaire des Institutions et de la Communication Intellectualles au Moyen Âge [CIVICIMA]. Études sur le vocabulaire intellectual du Moyen Âge, X, Brepols: Turnhout-Belgique, 2003.

ToYnBeE, Paget. Dante's Remarks on Translation in Convivio (i. 7, 11. 91-103). In: Toynbee, P. Dante Studies. Oxford University Press: Oxford, 1921.

Abreviaturas adoptadas

Conv. $=$ Convivio

De vulg. eloq. $=$ De vulgari eloquentia 